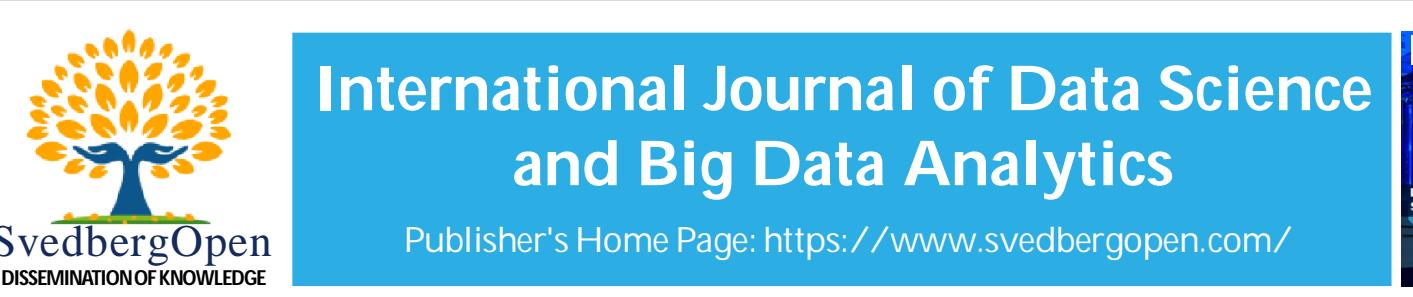

\title{
Generalized Least-Squares Ftting with Procedures for Uncorrelated Data of Constant Variance
}

Joachim W. Walewski ${ }^{*}$ and Thomas Metz ${ }^{2}$

'Division of Combustion Physics, Lund University, P.O. Box 118221 00, Lund, Sweden. E-mail: joachim.walewski@gmail.com ${ }^{2}$ Trimble Jena GmbH, Carl-Zeiss-Promenade 10, 07745 Jena, Germany.

Article Info

Volume 1, Issue 3, November 2021

Received : 25 March 2021

Accepted : 21 September 2021

Published : 05 November 2021

doi: $10.51483 / I J D S B D A .1 .3 .2021 .1-5$

\section{Abstract}

We show how generalized least-squares fitting, namely, the fitting of correlated data, can be carried out with algorithms for uncorrelated data of constant variance. Doing so requires only a simple linear transformation of the measurement data.

Keywords: Constant variance, Correlated data, Curve fitting, Generalized least-squares fitting, Least squares, Least-squares fitting, Maximum-likelihood estimation, Ordinary leastsquares fitting

(C) 2021 Joachim W. Walewski and Thomas Metz. This is an open access article under the CC BY license (https://creativecommons.org/licenses/by/4.0/), which permits unrestricted use, distribution, and reproduction in any medium, provided you give appropriate credit to the original author(s) and the source, provide a link to the Creative Commons license, and indicate if changes were made.

\section{Introduction}

Least-squares fitting of data is a recurring task in statistics and natural science. While there are many approaches and algorithms for performing least-squares fitting of uncorrelated data, only very few exist that support least-squares fitting correlated measurement data. This is not a major problem though, since-as we show in this application noteleast-squares fitting of correlated measurement data can be translated into a least-squares fitting problem for uncorrelated data of constant variance. The only thing needed is a simple linear transformation of the data.

\section{The Optimization Problem}

Least-squares fitting can be described as follows. The relationship between the varaibles $x_{1}, x_{2}, \ldots x_{m}$ is described by a model function.

$$
x=f(x, \alpha)
$$

where $x$ is a column vector of the $x$-values and $\alpha$ is the vector of the model parameters. An example for $f$ is

$$
x=\left(\begin{array}{l}
x_{1} \\
x_{2} \\
x_{3}
\end{array}\right)=\left(\begin{array}{c}
x_{1} \\
x_{2} \\
\alpha_{0}+\alpha_{1} x_{1}+\alpha_{2} x_{2}
\end{array}\right)
$$

where $f$ has two input variables $x_{1}$ and $x_{2}$, and one output variable $x_{3}$. This example is illustrated in Figure 1.

\footnotetext{
* Corresponding author: Joachim W. Walewski, Division of Combustion Physics, Lund University, P.O. Box 118221 00, Lund, Sweden.. E-mail: joachim.walewski@gmail.com
} 
In this application note, we assume that the $x$-values are complex. However, our findings also hold for real-valued $x$ 's.

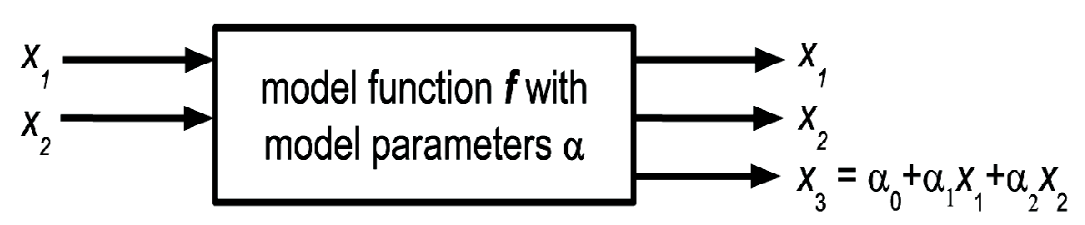

Figure 1: Illustration of the Model Function in Equation (2)

The problem to be solved is that the true $x$ - and $\alpha$-values are unknown. For instance, the $x$ values can only be observed with additive noise. Generalized least-squares curve fitting provides a way of estimating the true values. The estimates are written as $\hat{x}$ and $\hat{\alpha}$ and they are inferred by minimizing Equation (3).

$$
\chi^{2}=\delta^{\dagger} C^{-1} \delta
$$

where

$$
\delta:=x-f(\hat{x}, \hat{\alpha})
$$

The elements of the matrix $C$ are defined as:

$$
C_{i j}:=E\left(\left\{x_{i}-\varepsilon\left(x_{i}\right)\right\}\left\{x j-E\left(x_{j}\right)\right\}^{*}\right)
$$

for $1 \leq i, j \leq m$ [see, for instance (Taupin, 1988)]. $\varepsilon$ stands for the expectation value. The minimization of Equation (3) is referred to as generalized least-squares fitting.

Note that the $x$-values in Equation (5) are modeled as stochastic variables. For more details on observed values and stochastic variables see Appendix A.

Also note that $C_{i i}$ is the variance of $x_{i}$ and that $C_{i j}$ for $i \neq j$ indicates the correlation between $x_{i}$ and $x_{j}$. Furthermore, note that if $f$ is non-linear in $x, f$ may have to be replaced with a slightly modified function. For more details on this see (Metz et al., 2003).

While many analytical formulas and numerical procedures exist for the minimization of $\chi^{2}$ for uncorrelated $x$-values $\left(C_{i j}=0\right.$ for $\left.i \neq j\right)$, few exist for correlated $x$-values. This is especially true for commercially available procedures, which only seem to tackle the minimization of $\chi^{2}$ for uncorrelated $x$ values with constant variance. In other words, they only minimize

$$
\chi^{2}=\delta^{\dagger} \delta
$$

The minimization of Equation (6) is referred to as ordinary least-squares fitting.

The next section discusses how generalised least-squares fitting problems can be transformed into ordinary least-squares fitting problems.

\section{Translating Generalized Least-Squares Fitting into Ordinary Least-Squares Fitting}

This transformation exploits the fact that $C$ is hermitian [namely $C^{\dagger}=C$ according to Equation (5)]. See also (Wikipedia Contributors, 2021). Therefore, $C$ can be factored into:

$$
C=U V U^{\dagger}
$$

where $U$ and $V$ are constructed as follows (Wikipedia Contributors, 2020): solve the eignenvalue equation

$$
C u=v u
$$


The solutions are the vectors $u_{1}, u_{2}, \ldots u_{m}$ and the real values $v_{1}, v_{2} \ldots v_{m}$. Thereafter, construct $U$ according to $U=\left(u_{1}, u_{2}, \ldots u_{m}\right)$

and $V$ according to

$$
V_{i j}:=\left\{\begin{array}{l}
v_{i} \text { for } i=j \\
0 \text { for } i \neq j
\end{array}\right.
$$

Note that $U$ is a unitary matrix, meaning $U^{-1}=U^{\dagger}$ (Wikipedia Contributors, 2020).

$$
\delta=U \sigma \delta^{\prime}
$$

with

$$
\sigma_{i j}:=\left\{\begin{array}{c}
\sqrt{v_{i}} \text { for } i=j \\
0 \text { for } i \neq j
\end{array}\right.
$$

transforms Equation (3) into Equation (6). To demonstrate this, we insert Equation (11) into Equation (3):

$$
\begin{aligned}
\chi^{2} & =\delta^{\dagger} C^{-1} \delta \\
& =\left(U \sigma \delta^{\prime}\right)^{\dagger} C^{-1}\left(\mathrm{U} \sigma \delta^{\prime}\right) \\
& =\left(\delta^{\prime}\right)^{\dagger} \sigma^{\dagger} U^{\dagger} C^{-1} U \sigma \delta^{\prime}
\end{aligned}
$$

Using Equation (7), this yields'

$$
\begin{aligned}
\chi^{2} & =\left(\delta^{\prime}\right)^{\dagger} \sigma U^{\dagger}\left(U V U^{\dagger}\right)^{-1} U \sigma \delta^{\prime} \\
& =\left(\delta^{\prime}\right)^{\dagger} \sigma \underbrace{U^{\dagger}\left(U^{\dagger}\right)^{-1}}_{1} V^{-1} \underbrace{U^{-1} U}_{1} \sigma \delta^{\prime} \\
& =\left(\delta^{\prime}\right)^{\dagger} \sigma V^{-1} \sigma \delta^{\prime}
\end{aligned}
$$

where 1 is the identity matrix.

Remembering that $V=\sigma \sigma \quad$ [see Equation (12)], we get

$$
\begin{aligned}
\chi^{2} & =\left(\delta^{\prime}\right)^{\dagger} \underbrace{\sigma \sigma^{-1}}_{1} \underbrace{\sigma^{-1} \sigma}_{1} \delta^{\prime} \\
& =\left(\delta^{\prime}\right)^{\dagger} \delta^{\prime}
\end{aligned}
$$

which indeed is isomorphic to Equation (6) (Wikipedia Contributors, 2021).

\section{How to Use this Result in Practice?}

From Equation (11) and Equation (4), we get

$$
\begin{aligned}
& \delta^{\prime}=\sigma^{-1} U^{-1} \delta \\
& =\sigma^{-1} U^{-1} x-\sigma^{-1} U^{-1} f(\hat{x}, \hat{\alpha}) \\
& =\underbrace{\sigma^{-1} U^{-1} x}_{x^{\prime}}-\underbrace{\sigma^{-1} U^{-1} f(\hat{x}, \hat{\alpha})}_{f^{\prime}\left(\hat{x}^{\prime}, \hat{\alpha}^{\prime}\right)}
\end{aligned}
$$

The last row exploits the fact that $\delta^{\prime}=x^{\prime}-f^{\prime}\left(\hat{x}^{\prime}, \hat{\alpha}\right)$.

From Equation (16), the following recipe for fitting correlated data with algorithms for ordinary least-squares fitting can be inferred.

1. Take your measurement data $x$ and calculate

$$
x^{\prime}=\sigma^{-1} U^{\dagger} X
$$




\section{Calculate a new model function}

$$
f^{\prime}\left(x^{\prime}, \alpha\right)=\sigma^{-1} U^{\dagger} f\left(U \sigma \hat{x}^{\prime}, \alpha\right)
$$

3. Minimize

$\left(\delta^{\prime}\right)^{\dagger} \delta^{\prime}$

with

$\delta^{\prime}=x^{\prime}-f^{\prime}\left(x^{\prime}, \alpha\right)$

This step yields the estimates $\hat{x}^{\prime}$ and $\hat{\alpha}$

4. Calculate the estimates $\hat{x}$ with

$\hat{x}=U \sigma \hat{x}^{\prime}$

\section{Conclusion}

This application note provides a simple recipe for how to translate generalized least-squares curve fitting into leastsquares curve fitting of uncorrelated data with constant variance.

\section{Acknowledgment}

The authors thank the Center for Combustion Science and Technology, CE-COST, for financial support.

\section{References}

Metz, T., Walewski, J., and Kaminski, C.F. (2003). Maximum-likelihood Curve Fitting Scheme for Experiments with Pulsed Lasers Subject to Intensity Fluctuations. Applied Optics, 42, 1551-1563.

Taupin, D. (1988). Probabilities, Data Reduction and Error Analysis in the Physical Sciences. Les editions de physique, Les Ulis.

Wikipedia Contributors. (2020). Eigendecomposition of a Matrix-Wikipedia, the Free Encyclopedia [Online at https:// en.wikipedia.org/w/index.php?title=Eigendecomposition_of_a_matrix\&oldid=1012755753; accessed 2021-03-20].

Wikipedia Contributors. (2021a). Hermitian Matrix_-Wikipedia, the Free Encyclopedia [Online at https://en.wikipedia.org/ w/index.php?title=Hermitian_matrix\&oldid=999254083; accessed 11-January-2021].

Wikipedia Contributors. (2021b). Isomorphism—Wikipedia, the Free Encyclopedia [Online at https://en.wikipedia.org/ w/index.php?title=Isomorphism\&oldid=1007734513; accessed 20-March-2021]. 


\section{Appendix}

\section{Stochastic Variables and Observations}

The reader should be aware of the distinction between stochastic variables and observations. Stochastic variables are a mathematical concept for representing quantities that are subject to random variation. Stochastic variables are associated with a probability law, a so-called distribution function, and one can assign expectation values, variances, co-variances and so on to them.

Observations, on the other hand, are concrete numbers that are observed in, for instance, measurements, and they are typed into, say, a computer when performing some kind of evaluation.

Many textbooks on statistical inference use capital letters such as $X$ for stochastic variables and small letters such as $x$ for observations. In this work, we do not make this formal distinction in order to keep the formalism simple.

From the context, it will be clear what is meant. For instance, expectation values, variances etc. are only defined for stochastic variables, whereas a minimization of Equation (6) can only be done for concrete numbers, namely, the observations $x$.

Statistical inference is about estimating the values of unknown quantities. These estimates are obtained from the data of an experiment, so they are numbers you calculate from the observations.

Cite this article as: J oachim W. Wal ewski and Thomas Metz (2021). General ized Least-Squares Ftting with Procedures for U ncorrelated Data of Constant Variance. Devel oping an A I gorithmic Culture International Journal of D ata Scienceand Big D ata A nalytics, 1(3), 1-5. doi: 10.51483/ IJDSBD A .1.3.2021.1-5. 